\title{
Using Mobile Technology to Help Eliminate Malaria in Zanzibar
}

\author{
Gordon M. Cressman*1, Michael V. McKay ${ }^{1}$, Abdul-wahid Al-Mafazy², Mahdi M. Ramsan¹, \\ Abdullah S. Ali ${ }^{2}$, Issa A. Garimo' ${ }^{1}$, Humphrey Mkali² and Jeremiah J. Ngondi ${ }^{1}$
}

${ }^{1}$ RTI International, Research Triangle Park, NC, USA; 'Zanzibar Malaria Elimination Programme, Zanzibar Town, United Republic of Tanzania

\section{Objective}

This presentation aims to share the results of a six-year effort to use mobile health (mHealth) technology to help eliminate malaria from a well-defined geographic area. This presentation will review the history, technology, results, lessons-learned, and applicability to other contexts.

\section{Introduction}

Zanzibar is comprised primarily of two large islands with a population of 1.3 million. Indoor Residual Spraying (IRS) campaigns, distribution of long-lasting insecticide treated bed nets (LLINs), ensuring treatment medication is available, and use of Rapid Diagnostic Tests (RDTs) have reduced Malaria prevalence from $39 \%$ in 2005[1] to less than $1 \%$ in 2011-2012. This is the third time Zanzibar has been close to eliminating malaria, but there are serious challenges. These include vector resistance to pyrethroids, the shortlived efficacy of LLINs, and resistance to behavior change. Constant traffic with mainland Tanzania and foreign countries also poses the risk of outbreaks. An effective and sustained surveillance and rapid response system is essential to control outbreaks and optimize interventions.

\section{Methods}

In 2008 The Zanzibar Malaria Elimination Program (ZAMEP), in collaboration with the U.S. President's Malaria Initiative (PMI), the U.S. Centers for Disease Control and Prevention (CDC), and RTI International (RTI), developed and implemented the Malaria Epidemic Early Detection System (MEEDS). MEEDS enabled 159 government primary health care units to use simple forms displayed on feature phone handsets to submit weekly aggregate case data via Unstructured Supplementary Service Data (USSD). This enabled ZAMEP to make data-driven decisions regarding active case detection and coverage of malaria interventions.

In 2012 MEEDS was modified to support individual case reporting. Each District Malaria Surveillance Officer (DMSO) was equipped with a tablet computer running Coconut Surveillance, a mobile application developed by RTI in collaboration with ZAMEP. Coconut Surveillance receives case alerts from MEEDS automatically. Once a DMSO is alerted of a new case, he or she is guided through an active case response protocol by Coconut Surveillance. Additional case data are entered into the tablet at the facility and the household. Coconut Surveillance uses the Geographical Positioning System (GPS) capability of the tablet to record the location of the household. Each household member is tested, and new cases are treated immediately. The data are synchronized at least daily with a shared cloud database. A web-based dashboard enables near real-time monitoring of active follow-up. An automated notification system helps to alert officials to system errors and problems with case follow-up. Officials use near real-time maps and reports to quickly identify hot-spots and transmission patterns.

\section{Results}

Since January 2013 this integrated system has been used to respond to more than 4,000 case reports, Rapid Diagnostic Tests (RDTs) have been administered to and data collected from more than 14,400 household members, and 914 new cases have been diagnosed and treated. This provides Zanzibar with near real-time case identification, geo-located data, and management information about field staff progress. Continuous enhancement has added intelligence and automation to improve data quality, detect outbreaks, and alert decision makers.

\section{Conclusions}

The integrated MEEDS and Coconut Surveillance system in Zanzibar is recognized as one of the most advanced in the world, but it faces several challenges. For example, services for the MEEDS facility-based case reporting system are costly and, at times, unreliable. The system is not linked to the Health Management Information System (HMIS). Finally, cases from the extensive private sector, migrants and the military are not captured, and the system does not capture information on intervention quality.

\section{Keywords}

Malaria; Surveillance system; Surveillance-response systems; Mobile technology; Tanzania

\section{References}

1. Jaenisch T, Sullivan DJ, Dutta A, Deb S, Ramsan M, Othman MK, Gaczkowski R, Tielsch J, Sazawal S. Malaria incidence and prevalence on Pemba Island before the onset of the successful control intervention on the Zanzibar Archipelago. Malar J. 2010;7:32.

\section{*Gordon M. Cressman}

E-mail: gmc@rti.org 